\title{
On Superconformal Field Theories Associated to Very Attractive quartics
}

\author{
Katrin Wendland \\ University of Warwick, Gibbet Hill, Coventry CV4-7AL, England / UNC Chapel \\ Hill, CB\#3250 Phillips Hall, Chapel Hill, NC 27599-3250, USA \\ wendland@maths. warwick.ac.uk \\ Summary. We study $N=(4,4)$ superconformal field theories with left and right \\ central charge $c=6$ which allow geometric interpretations on specific quartic hy- \\ persurfaces in $\mathbb{C P}^{3}$. Namely, we recall the proof that the Gepner model $(2)^{4}$ admits \\ a geometric interpretation on the Fermat quartic and give an independent cross- \\ check of this result, providing a link to the "mirror moonshine phenomenon" on \\ K3. We clarify the rôle of Shioda-Inose structures in our proof and thereby gener- \\ alize it: We introduce VERY ATTRACTIVE QUARTICS and show how on each of them \\ a superconformal field theory can be constructed explicitly.
}

1 Moduli spaces associated to Calabi-Yau two-folds ........ 3

1.1 Complex structures .......................... 4

1.2 Hyperkähler structures . ........................ 5

$1.3 N=(4,4)$ Superconformal field theories ................ 6

2 Geometric interpretation of the Gepner model $(2)^{4} \ldots \ldots$. 8

3 A cross-check from physics: Phases on $K 3 \ldots \ldots \ldots \ldots \ldots 11$

$4 \quad$ An application of Shioda-Inose structures? . . . . . . . . . 15

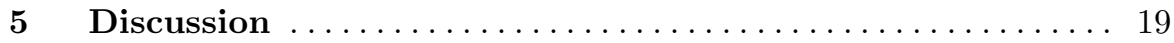

References..................................... 20

\section{Introduction}

Since the discovery of mirror symmetry [LVW89; COGP91; GP90] the quintic hypersurface in $\mathbb{C P}^{4}$ has presumably become the most prominent CalabiYau manifold in theoretical physics. Its two-dimensional relative, the Fermat quartic in $\mathbb{C P}^{3}$, has somewhat eluded such fame. However, the study of twodimensional Calabi-Yau manifolds seems rather promising from a conformal field theoretic point of view: On the one hand, the moduli space $\mathcal{M}_{S C F T}$ 
of superconformal field theories (SCFTs) associated to Calabi-Yau two-folds can be defined and studied on the level of abstract SCFTs, providing a sound basis for a mathematical analysis. On the other hand, the algebraic structure of $\mathcal{M}_{S C F T}$ is known explicitly, and its very description allows to draw links between geometry and SCFTs. Finally, to date only theories in fairly low dimensional subvarieties of $\mathcal{M}_{S C F T}$ have been constructed explicitly, rendering $\mathcal{M}_{S C F T}$ a non-trivial object to study.

In this note we investigate the Fermat quartic, and more generally so-called VERY ATTRACTIVE QUARTICS, from a SCFT point of view. The main ideas arise as applications of number theory and geometry to the study of SCFTs.

We start by giving an overview on the structure of the moduli space $\mathcal{M}_{S C F T}$ and its geometric predecessors. In particular, we discuss ATTRACTIVE and VERY ATTRACTIVE surfaces (Defs. 1, 2), providing a first link to arithmetic number theory. Along the way the "standard torus" $T_{0}$ and the Fermat quartic $X_{0} \subset \mathbb{C P}^{3}$ serve as our favorite examples. To discuss SCFTs associated to $X_{0}$, in Sect. 2 we recall our proof [NW01, Thm.2.13, Cor.3.6] that the Gepner model $(2)^{4}$ admits a geometric interpretation on $X_{0}$, i.e. with the same complex structure as $X_{0}$. We explain how the so-called SHioda-InoseSTRUCTURES give a guideline for the proof and how orbifold constructions allow us to also determine the normalized Kähler class, the volume, and the $B$-field of that geometric interpretation. In Sect. 3 we give an independent cross-check of these results by a careful application of Witten's analysis of phases in supersymmetric gauge theories [Wit93] to the $K 3$-case. We find that the Fricke MODUlar GROUP $\Gamma_{0}(2)_{+}$makes a natural appearance, providing a link to the arithmetic properties of the mirror map [NS95; LY96]. Sect. 4 is devoted to possible generalizations of our proof [NW01, Thm.2.13, Cor.3.6]. First, the rôle of Shioda-Inose structures is clarified. Then, for every very attractive quartic $X$ we find an $N=(4,4)$ SCFT $\mathcal{C}_{X}$ with $c=6$ which admits a geometric interpretation on $X$. In fact, $\mathcal{C}_{X}$ is a $\mathbb{Z}_{4}$ orbifold of a toroidal SCFT. As opposed to the known $\mathbb{Z}_{2}$ orbifold CFTs with geometric interpretation on $X$, we conjecture that $\mathcal{C}_{X}$ has a geometric interpretation with the full hyperkähler structure given by the natural one on $X \subset \mathbb{C P}^{3}$. We give evidence in favor of our conjecture, if (as implied by [Wit93]) it holds for $(2)^{4}$. We end with a discussion in Sect. 5 and state some open problems and implications, if in Sect. 4 we have indeed found "very attractive SCFTs", in general.

\section{Acknowledgments}

It is a pleasure to thank Paul Aspinwall, Gavin Brown, Gregory Moore, Werner Nahm, and Emanuel Scheidegger for helpful discussions. This note is a clarification and extension of ideas contained in our paper [NW01], and we wish to thank Werner Nahm for that collaboration. 


\section{Moduli spaces associated to Calabi-Yau two-folds}

We are interested in unitary, two-dimensional SCFTs with central charge $c=6$ which arise in string theory. These theories are expected to have nonlinear sigma model realizations on Calabi-Yau manifolds of complex dimension 2, i.e. either on a complex two-torus $T=Y^{\delta=0}$ or on a $K 3$-surface $X=Y^{\delta=16}$. In this note, $T, X, Y^{\delta}(\delta \in\{0,16\})$ will always denote the respective diffeomorphism type of a real four-manifold, with all additional structure to be chosen later. Recall the signature $\tau$ and Euler characteristic $\chi$ of these surfaces,

$-\tau\left(Y^{\delta=0}=T\right)=0=\delta, \quad \chi(T)=0, \quad-\tau\left(Y^{\delta=16}=X\right)=16=\delta, \quad \chi(X)=24$,

and the respective cohomology groups. They are equipped with the metric

$$
\alpha, \beta \in H^{*}\left(Y^{\delta}, \mathbb{R}\right): \quad\langle\alpha, \beta\rangle=\int_{Y^{\delta}} \alpha \wedge \beta,
$$

which is induced by the intersection form on homology under Poincaré duality:

$$
\delta \in\{0,16\}: \quad\left\{\begin{array}{l}
\mathbb{R}^{3,3+\delta} \cong H^{2}\left(Y^{\delta}, \mathbb{R}\right) \supset H^{2}\left(Y^{\delta}, \mathbb{Z}\right) \cong \Gamma^{3,3+\delta} \\
\mathbb{R}^{4,4+\delta} \cong H^{\text {even }}\left(Y^{\delta}, \mathbb{R}\right) \supset H^{\text {even }}\left(Y^{\delta}, \mathbb{Z}\right) \cong \Gamma^{4,4+\delta}
\end{array}\right.
$$

Here, $\Gamma^{p, q}$ denotes the standard even unimodular lattice of signature $(p, q)$, and the choice of the above isomorphisms amounts to the choice of a marking.

Example 0. On our STANDARD TORUs $T_{0}:=\mathbb{R}^{4} / \mathbb{Z}^{4}$ we use real Cartesian coordinates $x_{1}, \ldots, x_{4}$. A complex structure is introduced by choosing complex coordinates,

$$
T_{0}:=\mathbb{R}^{4} / \mathbb{Z}^{4}, \quad z_{1}:=x_{1}+i x_{2}, \quad z_{2}:=x_{3}+i x_{4}, \quad z_{k} \sim z_{k}+1 \sim z_{k}+i .
$$

Our STANDARD $K 3$-SURFACE is the FERMAT QUARTIC

$$
X_{0}: \quad z_{0}^{4}+z_{1}^{4}+z_{2}^{4}+z_{3}^{4}=0 \quad \text { in } \mathbb{C P}^{3}
$$

with the induced complex structure.

The pre-Hilbert space of a SCFT associated to a Calabi-Yau two-fold provides a representation of the $N=(4,4)$ superconformal algebra at $c=6$ which contains a left and a right handed Kac-Moody algebra $\mathfrak{s u}(2)_{l} \oplus \overline{\mathfrak{s u}(2)}{ }_{r}$, such that all charges with respect to a Cartan subalgebra (i.e. all doubled spins) are integral. Although explicit constructions are known only for a small number of theories associated to $K 3$, the moduli space of such SCFTs has been determined to a high degree of plausibility [Nar86; Sei88; AM94; NW01]. It should be compared to the known "classical" moduli spaces of geometric structures on Calabi-Yau two-folds. This section gives a summary of the relevant results, most of which can be found in [AM94; Asp97; NW01; Wen01; Wen02]. The mathematical background is beautifully explained in [BPdV84; Asp97]. 


\subsection{Complex structures}

The choice of a complex structure on a Calabi-Yau two-fold $Y^{\delta}$ is equivalent to the choice of a holomorphic volume form $\mu \in H^{2}\left(Y^{\delta}, \mathbb{C}\right)$ with $\mu \wedge \mu=0$, $\mu \wedge \bar{\mu}>0$. The real and imaginary part $\Omega_{1}, \Omega_{2} \in H^{2}\left(Y^{\delta}, \mathbb{R}\right)$ of $\mu$ hence span an oriented positive definite two-plane

$$
\Omega:=\operatorname{span}_{\mathbb{R}}\left\{\Omega_{1}, \Omega_{2}\right\} \subset H^{2}\left(Y^{\delta}, \mathbb{R}\right) \cong \mathbb{R}^{3,3+\delta} .
$$

In fact, by the Torelli theorem, there is a 1: 1 correspondence between such two-planes and points in the moduli space $\mathcal{M}_{c s}^{\delta}$ of complex structures on $Y^{\delta}$. To describe $\mathcal{M}_{c s}^{\delta}$, we fix a marking $H^{2}\left(Y^{\delta}, \mathbb{Z}\right) \cong \Gamma^{3,3+\delta}$ and express each two-plane $\Omega$ in terms of $H^{2}\left(Y^{\delta}, \mathbb{Z}\right)$. This gives a parametrization by the Grassmannian*

$$
\widetilde{\mathcal{M}}_{c s}^{\delta}=\mathrm{O}^{+}\left(H^{2}\left(Y^{\delta}, \mathbb{R}\right)\right) /(\mathrm{O}(2) \times \mathrm{O}(1,3+\delta))^{+} .
$$

The dependence on the marking is eliminated by dividing out the appropriate discrete group:

$$
\mathcal{M}_{c s}^{\delta}=\mathrm{O}^{+}\left(H^{2}\left(Y^{\delta}, \mathbb{Z}\right)\right) \backslash \mathrm{O}^{+}\left(H^{2}\left(Y^{\delta}, \mathbb{R}\right)\right) /(\mathrm{O}(2) \times \mathrm{O}(1,3+\delta))^{+} .
$$

For the standard torus $T_{0}$ as in (1), the holomorphic volume form is $\mu_{T_{0}}=$ $d z_{1} \wedge d z_{2}$, i.e.

$$
\Omega_{T_{0}}=\operatorname{span}_{\mathbb{R}}\left\{\Omega_{1}=d x_{1} \wedge d x_{3}+d x_{4} \wedge d x_{2}, \Omega_{2}=d x_{1} \wedge d x_{4}+d x_{2} \wedge d x_{3}\right\},
$$

such that $\Omega_{T_{0}}$ is generated by lattice vectors $\Omega_{k} \in H^{2}\left(T_{0}, \mathbb{Z}\right)$. Hence in this specific example, the NÉRON-SEVERI GROUP $\mathrm{NS}\left(T_{0}\right)=\Omega_{T_{0}}^{\perp} \cap H^{2}\left(T_{0}, \mathbb{Z}\right)$, which for Calabi-Yau two-folds agrees with the PICARD GROUP, has maximal rank $\rho\left(T_{0}\right)=6-2=4$. That is, $T_{0}$ is an ATTRACTIVE abelian variety:

Definition 1. Let $Y$ denote a Calabi-Yau two-fold with complex structure given by $\Omega_{Y} \subset H^{2}(Y, \mathbb{R})$ such that $Y$ has maximal PICARD NUMBER $\rho(Y):=$ $\operatorname{rk}(\operatorname{Pic}(Y)), \operatorname{Pic}(Y)=\operatorname{NS}(Y)=\Omega_{Y}^{\perp} \cap H^{2}(Y, \mathbb{Z})$. In other words, assume $\rho(Y)=\operatorname{rk}\left(H^{2}(Y, \mathbb{Z})\right)-2$. Then the complex surface $Y$ (or its complex structure $\left.\Omega_{Y}\right)$ is called an ATTRACTIVE surface ${ }^{\star \star}$.

The following results make these surfaces so attractive for us:

\section{Theorem 1.}

1. [SM74] To every attractive complex structure $\Omega_{T} \subset H^{2}(T, \mathbb{R})$ on a real four-torus $T$ we associate the quadratic form $Q_{T}$ of the TRANSCENDENTAL LATTICE $\Omega_{T} \cap H^{2}(T, \mathbb{Z})$. This gives a $1: 1$ correspondence between attractive complex two-tori and $\mathrm{SL}_{2}(\mathbb{Z})$ equivalence classes of positive definite even integral quadratic forms.

* For an inner product space $W$ with signature $(p, q), \mathrm{O}^{+}(W)$ denotes the component of $\mathrm{O}(W)$ which contains $\mathrm{SO}(p) \times \mathrm{O}(q)$. For $G \subset \mathrm{O}(W), G^{+}:=G \cap \mathrm{O}^{+}(W)$.

$\star \star$ The mathematical literature dubs such surfaces SINGULAR, which may easily cause confusion. We therefore rather borrow the terminology from [Mooa; Moob]. 
2. [SI77] The same is true for attractive K3-surfaces.

This means that in order to specify the complex structure of an attractive Calabi-Yau two-fold $Y$, it suffices to state the quadratic form $Q_{Y}$ of the transcendental lattice. Thm. 1 establishes a deep connection between the geometry of Calabi-Yau two-folds and the classification of positive definite even integral quadratic forms, i.e. a connection between geometry and number theory, which we shall make continuous use of in this work.

Example 1. For the standard torus $T_{0}$ with complex structure (1) one checks $Q_{T_{0}}=\operatorname{diag}(2,2)$. For the Fermat quartic (2) we have

Theorem 2. [Ino76] The Fermat quartic $X_{0}$ given by (2) is attractive, with quadratic form $Q_{X_{0}}=\operatorname{diag}(8,8)$ on the transcendental lattice.

In fact, Thm. 2 shows that the Fermat quartic is VERY ATTRACTIVE:

Definition 2. An attractive K3-surface $X$ is VERY ATTRACTIVE if the associated quadratic form $Q_{X}$ obeys

$$
Q_{X}=\left(\begin{array}{cc}
8 a & 4 b \\
4 b & 8 c
\end{array}\right)
$$

for some $a, b, c \in \mathbb{Z}$.

\subsection{Hyperkähler structures}

Calabi-Yau manifolds are Kähler by definition. If $Y^{\delta}(\delta \in\{0,16\})$ is equipped with a complex structure $\Omega \subset H^{2}\left(Y^{\delta}, \mathbb{R}\right)$, then by the Calabi-Yau theorem there is a 1: 1 correspondence between KäHLER CLASSES $\omega \in \Omega^{\perp} \cap H^{2}\left(Y^{\delta}, \mathbb{R}\right)$, $\langle\omega, \omega\rangle>0$, and Kähler-Einstein metrics on $Y^{\delta}$. The real Einstein metric underlying a pair $(\Omega, \omega)$ as above is specified by the positive definite oriented three-plane $\Sigma:=\operatorname{span}_{\mathbb{R}}(\Omega, \omega) \subset H^{2}\left(Y^{\delta}, \mathbb{R}\right)$, up to the volume. Since by considering $\Sigma$ instead of $(\Omega, \omega)$ the quantity $\langle\omega, \omega\rangle$ becomes superfluous, we can use any positive multiple of $\omega$, and we call it a NORMALIZED KäHLER CLASS. Combining Calabi-Yau and Torelli theorem one has a 1:1 correspondence between positive definite oriented three-planes $\Sigma \subset H^{2}\left(Y^{\delta}, \mathbb{R}\right)$ and real Einstein metrics on $Y^{\delta}$ (including orbifold limits), up to the volume. Given an Einstein metric $g$ on $Y^{\delta}$, the corresponding three-plane $\Sigma_{g}$ specifies an $\mathbb{S}^{2}$ of compatible complex structures $\Omega \subset \Sigma_{g}$, i.e. a unique hyperkähler structure. In fact, the associated Hodge star operator $*_{g}$ acts as involution on $H^{2}\left(Y^{\delta}, \mathbb{R}\right)$, and $\Sigma_{g}$ can be obtained as the $*_{g}$-invariant part of $H^{2}\left(Y^{\delta}, \mathbb{R}\right)$. It should be kept in mind that to date there is no direct method available which allows to reconstruct $g$ from $\Sigma_{g}$.

The moduli space $\mathcal{M}_{h k}^{\delta}$ of hyperkähler structures on $Y^{\delta}$ is now obtained in complete analogy to the moduli space $\mathcal{M}_{c s}^{\delta}$ of complex structures, c.f. (3):

$$
\mathcal{M}_{h k}^{\delta}=\mathrm{O}^{+}\left(H^{2}\left(Y^{\delta}, \mathbb{Z}\right)\right) \backslash \mathrm{O}^{+}\left(H^{2}\left(Y^{\delta}, \mathbb{R}\right)\right) / \mathrm{SO}(3) \times \mathrm{O}(3+\delta) .
$$


Example 2. The hyperkähler structure for our standard torus (1) is specified by $\Sigma_{T_{0}}=\operatorname{span}_{\mathbb{R}}\left(\Omega_{T_{0}}, \omega_{T_{0}}\right)$ with attractive $\Omega_{T_{0}}$ as in Ex. 1 and

$$
i\left(d z_{1} \wedge d \bar{z}_{1}+d z_{2} \wedge d \bar{z}_{2}\right) \quad \sim d x_{1} \wedge d x_{2}+d x_{3} \wedge d x_{4}=: \omega_{T_{0}} .
$$

Note that $\omega_{T_{0}} \in \Omega_{T_{0}}^{\perp} \cap H^{2}\left(T_{0}, \mathbb{Z}\right)$ with $\left\langle\omega_{T_{0}}, \omega_{T_{0}}\right\rangle=2$.

Similarly, we specify the hyperkähler structure $\Sigma_{X_{0}}$ on the Fermat quartic (2) by choosing as normalized Kähler class the class $\omega_{F S}$ induced by the Fubini-Study metric on $\mathbb{C P}^{3}$. Then $\Sigma_{X_{0}}:=\operatorname{span}_{\mathbb{R}}\left(\Omega_{X_{0}}, \omega_{F S}\right)$ with attractive $\Omega_{X_{0}}$ as in Thm. 2, and $\omega_{F S} \in \Omega_{X_{0}}^{\perp} \cap H^{2}\left(X_{0}, \mathbb{Z}\right)$ with $\left\langle\omega_{F S}, \omega_{F S}\right\rangle=4$.

\section{3 $N=(4,4)$ Superconformal field theories}

By the above, the specification of a hyperkähler structure on a Calabi-Yau two-fold $Y^{\delta}(\delta \in\{0,16\})$ by a three-plane $\Sigma \subset H^{2}\left(Y^{\delta}, \mathbb{R}\right)$ is equivalent to the specification of a real Einstein metric $g$ on $Y^{\delta}$, up to the volume, where $\Sigma$ is the $*_{g}$-invariant subspace of $H^{2}\left(Y^{\delta}, \mathbb{R}\right)$. To incorporate the volume $V \in \mathbb{R}^{+}$, one may note that $*_{g}$ also acts as involution on $H^{\text {even }}\left(Y^{\delta}, \mathbb{R}\right)$ and consider the $*_{g}$-invariant subspace, there. But not every positive definite oriented fourplane $x \subset H^{\text {even }}\left(Y^{\delta}, \mathbb{R}\right)$ can be interpreted as $(+1)$-eigenspace of such a Hodge star operator. However, Aspinwall and Morrison [AM94] noticed that after the choice of a grading for $H^{\text {even }}\left(Y^{\delta}, \mathbb{R}\right) \cong \mathbb{R}^{4,4+\delta}$ into $H^{\text {even }}=H^{0} \oplus H^{2} \oplus H^{4}$ by selecting $\mathbb{Z}$-generators $v^{0}, v$ of $H^{0}\left(Y^{\delta}, \mathbb{Z}\right), H^{4}\left(Y^{\delta}, \mathbb{Z}\right)$, there exists a natural projection from the Grassmannian of all positive definite oriented four-planes in $H^{\text {even }}\left(Y^{\delta}, \mathbb{R}\right)$,

$$
\widetilde{\mathcal{M}}_{S C F T}^{\delta}:=\mathrm{O}^{+}\left(H^{\text {even }}\left(Y^{\delta}, \mathbb{R}\right)\right) / \mathrm{SO}(4) \times \mathrm{O}(4+\delta), \quad \delta \in\{0,16\},
$$

to the parameter space of Einstein metrics on $Y^{\delta}$ : With $H^{2}\left(Y^{\delta}, \mathbb{R}\right)=\left(v^{0}\right)^{\perp} \cap$ $v^{\perp} \cap H^{e v e n}\left(Y^{\delta}, \mathbb{R}\right)$,

$$
\begin{aligned}
& \widetilde{\mathcal{M}}_{S C F T}^{\delta} \longrightarrow \widetilde{\mathcal{M}}_{h k}^{\delta} \times \mathbb{R}^{+} \times H^{2}\left(Y^{\delta}, \mathbb{R}\right), \\
& x \longmapsto \quad(\Sigma, V \quad, B), \\
& x=\operatorname{span}_{\mathbb{R}}\left\{\xi(\Sigma), v^{0}+B+\left(V-\frac{1}{2}\langle B, B\rangle\right) v\right\}, \\
& \xi(\sigma):=\sigma-\langle\sigma, B\rangle v \text { for } \sigma \in H^{2}\left(Y^{\delta}, \mathbb{R}\right) .
\end{aligned}
$$

Here, $v^{0}, v$ can be characterized by

$$
v^{0}, v \in H^{\text {even }}\left(Y^{\delta}, \mathbb{Z}\right): \quad\left\langle v^{0}, v^{0}\right\rangle=\langle v, v\rangle=0, \quad\left\langle v^{0}, v\right\rangle=1 .
$$

Note that this gives $\widetilde{\mathcal{M}}_{S C F T}^{\delta}$ the structure of a bundle over the parameter space $\widetilde{\mathcal{M}}_{h k}^{\delta} \times \mathbb{R}^{+}$of Einstein metrics on $Y^{\delta}$. We interpret $V \in \mathbb{R}^{+}$as parameter of volume, and the fiber coordinate $B \in H^{2}\left(Y^{\delta}, \mathbb{R}\right)$ is known as the $B$-FIELD. In fact, the right hand side of (5) is the parameter space of nonlinear sigma models on $Y^{\delta}$. On the other hand, using the representation theory of our $N=$ 
$(4,4)$ superconformal algebra at $c=6$ and deformation theory of SCFTs, one shows that each component of the parameter space of $N=(4,4)$ SCFTs with $c=6$ is isomorphic to a Grassmannian (4), see [Nar86; Sei88; Cec91; AM94]. Finally, there is a natural warped product metric on the right hand side of (5) which allows to interpret that map as isometry between an irreducible component of the parameter space of $N=(4,4)$ SCFTs at $c=6$, equipped with the Zamolodchikov metric, and the parameter space of nonlinear sigma models on $Y^{\delta}$. Thus this interpretation is compatible with the deformation theory both of SCFTs on the left hand side and of the geometric data on the right hand side of (5) [AM94].

As for $\mathcal{M}_{c s}^{\delta}$ and $\mathcal{M}_{h k}^{\delta}$, the moduli space is obtained from its smooth universal cover $\widetilde{\mathcal{M}}_{S C F T}^{\delta}$ by dividing out a discrete group of lattice automorphisms [Nar86; AM94; NW01]:

$$
\mathcal{M}_{S C F T}^{\delta}=\mathrm{O}^{+}\left(H^{\text {even }}\left(Y^{\delta}, \mathbb{Z}\right)\right) \backslash \mathrm{O}^{+}\left(H^{\text {even }}\left(Y^{\delta}, \mathbb{R}\right)\right) / \mathrm{SO}(4) \times \mathrm{O}(4+\delta) .
$$

Summarizing, the moduli space of $N=(4,4)$ SCFTs at $c=6$ decomposes into two components ${ }^{\star \star \star} \mathcal{M}_{S C F T}^{\delta}, \delta \in\{0,16\}$, and every $N=(4,4)$ SCFT with $c=6$ can be associated either to $K 3$ or to the torus, depending on the value of $\delta$. Indeed, given an $N=(4,4) \operatorname{SCFT} \mathcal{C}$ with $c=6$ one can determine $\delta$ by calculating the conformal field theoretic elliptic genus of $\mathcal{C}$, which agrees with the geometric elliptic genus of $Y^{\delta}$. The theories in $\widetilde{\mathcal{M}}_{S C F T}^{\text {tori }}=\widetilde{\mathcal{M}}_{S C F T}^{0}$ are called TOROIDAL SCFTs. Each component $\widetilde{\mathcal{M}}_{S C F T}^{\delta}$ of the parameter space is a natural extension of the parameter space of Einstein metrics on $Y^{\delta}$, such that every oriented positive definite four-plane $x \subset H^{\text {even }}\left(Y^{\delta}, \mathbb{R}\right)$ corresponds to a $\mathrm{SCFT}^{\dagger}$. More precisely, (7) is a partial completion of the actual moduli space of SCFTs on $K 3$, see [Wit95]:

$$
\begin{aligned}
x & \in \mathrm{O}^{+}\left(H^{\text {even }}(X, \mathbb{R})\right) / \mathrm{SO}(4) \times \mathrm{O}(4+\delta) \quad \text { corresponds to a SCFT } \\
& \Longleftrightarrow x \subset H^{\text {even }}(X, \mathbb{R}) \text { s.th. }\left\{e \in x^{\perp} \cap H^{\text {even }}(X, \mathbb{Z}) \mid\langle e, e\rangle=-2\right\}=\emptyset .
\end{aligned}
$$

Each choice of generators $v^{0}, v$ of $H^{0}\left(Y^{\delta}, \mathbb{Z}\right), H^{4}\left(Y^{\delta}, \mathbb{Z}\right)$ with $(6)$ specifies a GEOMETRIC INTERPRETATION $(\Sigma, V, B)$ as in (5). The discrete group $\mathrm{O}^{+}\left(H^{\text {even }}\left(Y^{\delta}, \mathbb{Z}\right)\right)$ acts transitively on all possible pairs $\left(v^{0}, v\right)$ with $(6)$. If a SCFT $\mathcal{C}$ in $\mathcal{M}_{S C F T}^{\delta}$ has geometric interpretation $(\Sigma, V, B)$ such that $\Omega \subset \Sigma$ gives the complex structure of a surface $Y_{0}^{\delta}$ as in Ex. 1, then we say that $\mathcal{C}$ ADMITS A GEOMETRIC INTERPRETATION ON $Y_{0}^{\delta}$.

Example 3. It is easy to determine the location of every toroidal SCFT in $\mathcal{M}_{S C F T}^{\text {tori }}=\mathcal{M}_{S C F T}^{0}$ and to construct all theories in $\mathcal{M}_{S C F T}^{\text {tori } i}$ explicitly, see

$\star \star \star$ We can prove that the component $\mathcal{M}_{S C F T}^{0}$ is unique, but we cannot exclude the occurrence of multiple components $\mathcal{M}_{S C F T}^{16}$. However, to date no example of an $N=(4,4)$ SCFT with $c=6$ has been found to contradict uniqueness of $\mathcal{M}_{S C F T}^{16}$, and in the following we restrict attention to a single such component.

$\dagger$ By abuse of notation we generally denote a four-plane in $H^{\text {even }}\left(Y^{\delta}, \mathbb{R}\right)$ and the corresponding $x \in \mathrm{O}^{+}\left(H^{\text {even }}\left(Y^{\delta}, \mathbb{R}\right)\right) / \mathrm{SO}(4) \times \mathrm{O}(4+\delta)$ by the same symbol. 
[Nar86]. E.g., the torus (1) has volume $V_{T_{0}}=1$, and setting the $B$-field to zero, $B_{T_{0}}:=0$, we can construct a SCFT $\mathcal{T}_{0}$ corresponding to

$$
\mathcal{T}_{0}: \quad x_{T_{0}}:=\operatorname{span}_{\mathbb{R}}\left(\Omega_{T_{0}}, \omega_{T_{0}}, v^{0}+v\right) \in \widetilde{\mathcal{M}}_{S C F T}^{\text {tori }},
$$

with $\Omega_{T_{0}}, \omega_{T_{0}}$ as in Exs. 1, 2. On the other hand, it is not easy to determine the explicit location of an abstractly defined SCFT within $\mathcal{M}_{S C F T}^{K 3}$. Similarly, only very few theories in $\mathcal{M}_{S C F T}^{K 3}$ have been constructed explicitly, so far. As to the example of the Fermat quartic $X_{0}$, it has been conjectured [Gep87; Gep88] that the Gepner model $(2)^{4}$ should admit a geometric interpretation on $X_{0}$. Much evidence in favor of this conjecture has been collected, in particular [Wit93], and the following two sections are devoted to an explanation of its proof.

\section{Geometric interpretation of the Gepner model $(2)^{4}$}

In this section we give a summary of our proof [NW01, Cor.3.6] that the Gepner model $(2)^{4}$ admits a geometric interpretation on the Fermat quartic $X_{0}$. In fact, in [EOTY89] it was conjectured that $(2)^{4}$ agrees with the $\mathbb{Z}_{4}$ orbifold of our standard toroidal theory $\mathcal{T}_{0}$, see $(9)$, since the respective partition functions agree. Orbifold constructions have been studied in detail in [NW01; Wen01; Wen02], and the interested reader is referred to these papers for further explanations. Here, only the following result from [NW01; Wen01] is needed:

Proposition 1. Let $M \in\{2,3,4,6\}$, and let $T=\mathbb{R}^{4} / \Lambda$ denote a real fourtorus with Einstein metric $g$ which is $\mathbb{Z}_{M}$ symmetric. In other words, there are a complex structure and complex coordinates $\left(z_{1}, z_{2}\right)$ which are compatible with $g$ and such that $g$ is invariant under

$$
\zeta_{M}: \quad\left(z_{1}, z_{2}\right) \longmapsto\left(e^{2 \pi i / M} z_{1}, e^{-2 \pi i / M} z_{2}\right), \quad \zeta_{M} \Lambda=\Lambda,
$$

i.e. $\Sigma_{g} \subset H^{2}(T, \mathbb{R})^{\mathbb{Z}_{M}}$. Let $V_{T} \in \mathbb{R}^{+}$denote the volume of $T$ and $B_{T} \in$ $H^{2}(T, \mathbb{R})^{\mathbb{Z}_{M}}$ a B-field, specifying a toroidal SCFT $\mathcal{T}$. Then the $\mathbb{Z}_{M}$-action $\zeta_{M}$ extends to a symmetry of $\mathcal{T}$, and the corresponding orbifold CFT, denoted $\mathcal{T} / \mathbb{Z}_{M}$, has a geometric interpretation $(\Sigma, V, B)$ on the $\mathbb{Z}_{M}$ orbifold limit $X=$ $\widehat{T / \mathbb{Z}_{M}}$ of $K 3$, which is obtained from $T / \mathbb{Z}_{M}$ by minimally resolving all orbifold singularities. Let $\pi: T \longrightarrow X=\widetilde{T / \mathbb{Z}_{M}}$ denote the rational map obtained from the minimal resolution, then

$$
(\Sigma, V, B)=\left(\pi_{*} \Sigma_{g}, \frac{V_{T}}{M}, \frac{1}{M} \pi_{*} B_{T}+\frac{1}{M} \check{B}_{M}\right),
$$

where $\check{B}_{M} \in H^{2}(X, \mathbb{Z}) \cap\left(\pi_{*} H^{2}(T, \mathbb{Z})^{\mathbb{Z}_{M}}\right)^{\perp}$ is a fixed primitive lattice vector with $\left\langle\check{B}_{M}, \check{B}_{M}\right\rangle=-2 M^{2}$. 
The following application of Prop. 1 is a helpful exercise, see Sect. 4:

Corollary 1. Let $a, b, c \in \mathbb{Z}$ such that

$$
Q_{a, b, c}:=\left(\begin{array}{ll}
8 a & 4 b \\
4 b & 8 c
\end{array}\right)
$$

is positive definite. Then there is a toroidal SCFT $\mathcal{T}_{a, b, c}$ with $\mathbb{Z}_{4}$ orbifold $\mathcal{T}_{a, b, c} / \mathbb{Z}_{4}$ in $\mathcal{M}_{S C F T}^{K 3}$ corresponding to a four-plane $x_{a, b, c} \subset H^{\text {even }}(X, \mathbb{R})$ such that the following hold $s^{\ddagger}: x_{a, b, c}=\Omega^{*} \oplus \mho_{a, b, c}$, where $\Omega^{*}, \mho_{a, b, c}$ are two-planes in $H^{\text {even }}(X, \mathbb{R})$, such that $\Omega^{*} \cap H^{\text {even }}(X, \mathbb{Z}), \mho_{a, b, c} \cap H^{\text {even }}(X, \mathbb{Z})$ have rank 2 and quadratic forms $Q_{\Omega^{*}}=\operatorname{diag}(2,2)$ and $Q_{\mho_{a, b, c}}=Q_{a, b, c}$, respectively.

Proof. Consider $T=\mathbb{R}^{4} / \Lambda=E_{1} \times E_{2}$ with orthogonal real two-tori $E_{k}$ at radii $R_{k}, k \in\{1,2\}$. That is, with respect to real Cartesian coordinates $x_{1}, \ldots, x_{4}$ and the basis $d x_{1}, \ldots, d x_{4}$ of $H^{1}(T, \mathbb{R})$, the $\mathbb{Z}$-dual $\Lambda^{*} \cong H^{1}(T, \mathbb{Z})$ of $\Lambda$ is generated by $\mu_{1}, \ldots, \mu_{4}$ such that

$$
\exists R_{1}, R_{2} \in \mathbb{R}^{+}: \quad\left(\mu_{1}, \ldots, \mu_{4}\right)=\operatorname{diag}\left(R_{1}, R_{1}, R_{2}, R_{2}\right)^{-1} .
$$

We choose a complex structure by setting $z_{1}:=x_{1}+i x_{2}, z_{2}:=x_{3}+i x_{4}$, and a Kähler class $\omega_{R_{2}^{2} / R_{1}^{2}} \sim i\left(d z_{1} \wedge d \bar{z}_{1}+d z_{2} \wedge d \bar{z}_{2}\right)$ :

$$
\begin{aligned}
& d x_{1} \wedge d x_{3}+d x_{4} \wedge d x_{2} \sim \mu_{1} \wedge \mu_{3}+\mu_{4} \wedge \mu_{2}=: \Omega_{1}, \\
& d x_{1} \wedge d x_{4}+d x_{2} \wedge d x_{3} \sim \mu_{1} \wedge \mu_{4}+\mu_{2} \wedge \mu_{3}=: \Omega_{2}, \\
& d x_{1} \wedge d x_{2}+d x_{3} \wedge d x_{4} \sim \mu_{1} \wedge \mu_{2}+\frac{R_{2}^{2}}{R_{1}^{2}} \mu_{3} \wedge \mu_{4}=: \omega_{R_{2}^{2} / R_{1}^{2}}
\end{aligned}
$$

Here, $\Lambda$ and $\Sigma:=\operatorname{span}_{\mathbb{R}}\left(\Omega_{1}, \Omega_{2}, \omega_{R_{2}^{2} / R_{1}^{2}}\right)$ are invariant under $\zeta_{4}:\left(z_{1}, z_{2}\right) \longmapsto$ $\left(i z_{1},-i z_{2}\right)$, i.e. $\Sigma \subset H^{2}(T, \mathbb{R})^{\mathbb{Z}_{4}}$, and $V_{T}=R_{1}^{2} R_{2}^{2}$ is the volume of $T$. For $a, b, c \in \mathbb{Z}$ as in the claim, $a>0,4 a c-b^{2}>0$, since $Q_{a, b, c}$ is positive definite. Let

$$
\frac{R_{2}^{2}}{R_{1}^{2}}:=a, \quad V_{T}=R_{1}^{2} R_{2}^{2}:=c-\frac{b^{2}}{4 a}, \quad B_{T}:=-\frac{b}{2 a} \omega_{a} .
$$

These data specify a toroidal SCFT $\mathcal{T}_{a, b, c}$, and in terms of (5),

$$
x_{T}=\operatorname{span}_{\mathbb{R}}\left\{\Omega_{1}, \Omega_{2}, \omega_{a}-\left\langle\omega_{a}, B_{T}\right\rangle v, v^{0}+B_{T}+\left(V_{T}-\frac{1}{2}\left\langle B_{T}, B_{T}\right\rangle\right) v\right\},
$$

where $\left(\left\langle\Omega_{i}, \Omega_{j}\right\rangle\right)_{i, j}=\operatorname{diag}(2,2),\left\langle\omega_{a}, \omega_{a}\right\rangle=2 a$. By Prop. $1, \mathcal{T}_{a, b, c} / \mathbb{Z}_{4}$ is given by $x=\Omega^{*} \oplus \mho_{a, b, c} \subset H^{e v e n}(X, \mathbb{R})$, where with respect to appropriate generators $\widehat{v}^{0}, \widehat{v}$ of $H^{0}(X, \mathbb{Z}), H^{4}(X, \mathbb{Z})$

$$
\begin{aligned}
\Omega^{*}= & \operatorname{span}_{\mathbb{R}}\left\{\pi_{*} \Omega_{1}, \pi_{*} \Omega_{2}\right\}, \\
\mho_{a, b, c}= & \operatorname{span}_{\mathbb{R}}\left\{\xi_{1}:=\widetilde{\omega}_{a}-\left\langle\widetilde{\omega}_{a}, \widetilde{B}\right\rangle \widehat{v}, \xi_{0}:=\widehat{v}^{0}+\widetilde{B}+\left(\frac{V_{T}}{4}-\frac{1}{2}\langle\widetilde{B}, \widetilde{B}\rangle\right) \widehat{v}\right\} \\
& \text { with } \widetilde{\omega}_{a}:=\pi_{*} \omega_{a}, \quad \widetilde{B}:=\frac{1}{4} \pi_{*} B_{T}+\frac{1}{4} \check{B}_{4}=-\frac{b}{8 a} \widetilde{\omega}_{a}+\frac{1}{4} \check{B}_{4},
\end{aligned}
$$

$\ddagger$ To clear notations, we generally let $\oplus$ denote the orthogonal direct sum. 
and $\left\langle\breve{B}_{4}, \breve{B}_{4}\right\rangle=-32$. First, by the results of [SI77], $\Omega^{*}=\operatorname{span}_{\mathbb{R}}\left\{\widetilde{\Omega}_{1}, \widetilde{\Omega}_{2}\right\}$ with $\widetilde{\Omega}_{k}=\frac{1}{2} \pi_{*} \Omega_{k} \in H^{2}(X, \mathbb{Z})$. Since $\pi$ has degree 4 by construction, $\left(\left\langle\widetilde{\Omega}_{i}, \widetilde{\Omega}_{j}\right\rangle\right)_{i, j}=\operatorname{diag}(2,2)$ and $\left\langle\widetilde{\omega}_{a}, \widetilde{\omega}_{a}\right\rangle=4\left\langle\omega_{a}, \omega_{a}\right\rangle=8 a$, such that

$$
\begin{aligned}
& \mho_{a, b, c} \cap H^{\text {even }}(X, \mathbb{Z}) \\
& \quad=\operatorname{span}_{\mathbb{Z}}\left\{\xi_{1}=\widetilde{\omega}_{a}+b \widehat{v}, \xi_{2}:=4 \xi_{0}+\frac{b}{2 a} \xi_{1}=4 \widehat{v}^{0}+\check{B}_{4}+(c+4) \widehat{v}\right\}
\end{aligned}
$$

with $\left(\left\langle\xi_{i}, \xi_{j}\right\rangle\right)_{i, j}=Q_{a, b, c}$. This proves the claim.

Instead of giving a direct proof for the claim $(2)^{4}=\mathcal{T}_{0} / \mathbb{Z}_{4}$ of [EOTY89], we use the following result as a helpful guide, without applying it explicitly (see Sect. 4):

Theorem 3. [SI77] Let $X$ denote an attractive K3-surface, which by Thm. 1 is specified by its associated quadratic form $Q_{X}$. Then $X$ allows a symplectic $\mathbb{Z}_{2}$-action $\iota$ (that is, $\iota$ induces a trivial action on $\Omega_{X} \subset H^{2}(X, \mathbb{R})$ ) such that $Y=\widetilde{X / \iota}$ is attractive with associated quadratic form $Q_{Y}=2 Q_{X}$. Moreover, $Y=\widetilde{X / \iota}$ gives the Kummer SURFACE $\widetilde{T / \mathbb{Z}_{2}}$ of the attractive two-torus $T$ (see Prop. 1) with associated quadratic form $Q_{T}=Q_{X}$.

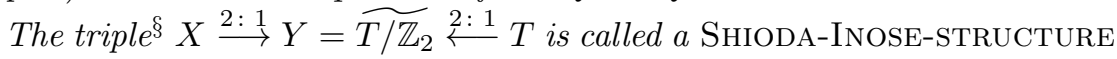
(in short an SI-STRUCTURE).

Now note that $X=\widetilde{T_{0} / \mathbb{Z}_{4}}$ is an attractive $K 3$-surface, which by the result of [SI77] mentioned in the proof of Cor. 1 has associated quadratic form $Q_{X}=$ $\operatorname{diag}(2,2)=Q_{T_{0}}$. Hence by Thm. 3 there is an SI-structure $X={\widetilde{T_{0}} / \mathbb{Z}_{4}}_{2: 1}^{\longrightarrow}$ $\widetilde{T_{0} / \mathbb{Z}_{2}} \stackrel{2: 1}{\stackrel{1}{2}} T_{0}$, and $Y=\widetilde{T_{0} / \mathbb{Z}_{2}}$ can be obtained from $X=\widetilde{T_{0} / \mathbb{Z}_{4}}$ by a $\mathbb{Z}_{2}$ orbifold construction. If $(2)^{4}=\mathcal{T}_{0} / \mathbb{Z}_{4}$, and if all symplectic symmetries extend to symmetries of the corresponding SCFTs, then we can expect a $\mathbb{Z}_{2}$ orbifold of $(2)^{4}$ to agree with $\mathcal{T}_{0} / \mathbb{Z}_{2}$. Indeed:

Proposition 2. [NW01, Thm.3.3] Let $\sigma:=[2,2,0,0]$ denote the Gepner phase symmetry which acts as the parafermionic $\mathbb{Z}_{2}$ on each of the first two factors of $(2)^{4}$. Then $(\widehat{2})^{4}:=(2)^{4} / \sigma$ agrees with $\mathcal{T}_{0} / \mathbb{Z}_{2}$.

It is now easy to find a $\mathbb{Z}_{2}$-action $\eta$ on $(\widehat{2})^{4}$ such that $(2)^{4}=(\widehat{2})^{4} / \eta$. Moreover, the proof of Prop. 2 gives an explicit dictionary between CFT and geometric data of $(\widehat{2})^{4}$ and $Y=\widetilde{T_{0} / \mathbb{Z}_{2}}$, respectively, which allows to show that $\eta$ is induced by a symplectic $\mathbb{Z}_{2}$-action $\eta$ on $Y$. One then checks that $\widetilde{Y / \eta}=$ $\widetilde{T_{0} / \mathbb{Z}_{4}}=X$, which together with Prop. 1 proves

Proposition 3. [NW01, Thm.3.5] The Gepner model $(2)^{4}$ has a geometric interpretation $\left(\pi_{*} \Sigma_{T_{0}}, V, B\right)$ on $X=\widetilde{T_{0} / \mathbb{Z}_{4}}$ with volume $V=\frac{1}{4}$ and $B$-field $B=\frac{1}{4} \check{B}_{4}$, where $\check{B}_{4} \in H^{2}(X, \mathbb{Z}) \cap\left(\pi_{*} H^{2}\left(T_{0}, \mathbb{Z}\right)^{\mathbb{Z}_{4}}\right)^{\perp}$ and $\left\langle\check{B}_{4}, \check{B}_{4}\right\rangle=-32$.

\footnotetext{
$\S$ Here and in the following, $\stackrel{n: 1}{\longrightarrow}$ and $\stackrel{n: 1}{\longleftarrow}$ denote rational maps of degree $n$.
} 
By Sect. 1.2, we can now write the four-plane $x_{(2)^{4}} \in \widetilde{\mathcal{M}}_{S C F T}^{K 3}$ that corresponds to $(2)^{4}$ in the form (5), using the data found in Prop. 3 . Then we determine a new pair $\left(v_{Q}^{0}, v_{Q}\right)$ of null vectors with (6) to rewrite $x_{(2)^{4}}$ :

$$
x_{(2)^{4}}=\operatorname{span}_{\mathbb{R}}\left\{\xi\left(\Sigma_{Q}\right), v_{Q}^{0}+B_{Q}+\left(V_{Q}-\frac{1}{2}\left\langle B_{Q}, B_{Q}\right\rangle\right) v_{Q}\right\} .
$$

If there is a two-plane $\Omega_{Q} \subset \Sigma_{Q}$ such that $\Omega_{Q} \cap H^{2}(X, \mathbb{Z})$ has rank 2 and quadratic form $Q_{X_{0}}=\operatorname{diag}(8,8)$, then Thms. 1, 2 show that $(2)^{4}$ admits a geometric interpretation on the Fermat quartic. The explicit form of (10) moreover allows us to determine the normalized Kähler class, the volume, and the $B$-field of this geometric interpretation. Indeed,

Proposition 4. [NW01, Thm.2.13, Cor.3.6] The Gepner model $(2)^{4}$ admits a geometric interpretation on the Fermat quartic $X_{0} \subset \mathbb{C P}^{3}$ with normalized Kähler class $\omega_{Q} \in H^{2}\left(X_{0}, \mathbb{Z}\right),\left\langle\omega_{Q}, \omega_{Q}\right\rangle=4$, volume $V=\frac{1}{2}$, and B-field $B=-\frac{1}{2} \omega_{Q}$.

Note that $\omega_{Q}$ may agree with the natural Kähler class $\omega_{F S}$ on $X_{0} \subset \mathbb{C P}^{3}$, $\left\langle\omega_{F S}, \omega_{F S}\right\rangle=4$, but the above methods do not prove this.

\section{A cross-check from physics: Phases on $K 3$}

Let us take a closer look at the origin of the conjecture that $(2)^{4}$ should admit a geometric interpretation on the Fermat quartic. We use Witten's analysis of linear sigma models [Wit93] and the assumption that under the renormalization group flow each such theory flows to a (maybe degenerate) SCFT at the infrared fixed point. This in particular implies that for every quartic hypersurface $X \subset \mathbb{C P}^{3}$, there should exist a family $\mathcal{F}_{X}=\left(\mathcal{C}_{w}, w=e^{r+i \vartheta} \in \mathbb{C} \cup\{\infty\}\right)$ of $N=(4,4)$ SCFTs at $c=6$ with geometric interpretation on $X$, normalized Kähler class $\omega_{F S}$ induced by the Fubini-Study metric on $\mathbb{C P}^{3}$, and $B$-field $B=\beta \omega_{F S}, \beta \in \mathbb{R}$. Moreover, the theory at $w=1$ violates (8), hence is not well-defined, whereas $w=\infty$ corresponds to a large volume limit of $X$. The family has maximal unipotent monodromy around $w=\infty$, monodromy of order 2 around $w=1$, and monodromy of order 4 around $w=0$. In fact, for $X=X_{0}, w=0$ should give the Gepner model $(2)^{4}$.

In the smooth universal covering space $\widetilde{\mathcal{M}}_{S C F T}^{K 3}$ (see (4)), the lift of each family $\mathcal{F}_{X}$ can be described within a fixed geometric interpretation, i.e. we once and for all choose null vectors $v^{0}, v$ that generate $H^{0}(X, \mathbb{Z}), H^{4}(X, \mathbb{Z})$ to use (5). Above, we have also specified a complex structure and a normalized Kähler class, or equivalently the three-plane $\Sigma$. We focus on the Fermat quartic $X_{0}$ in the following, so $\Sigma_{X_{0}}=\operatorname{span}_{\mathbb{R}}\left(\Omega_{X_{0}}, \omega_{F S}\right)$ as in Ex. 2. Moreover, $B=\beta \omega_{F S}$, which allows us to cast all four-planes in our family into the following form:

$$
\begin{gathered}
x=\Omega_{X} \oplus \mho_{\beta, V} \quad \text { where with } \quad\left\langle\omega_{F S}, \omega_{F S}\right\rangle=4, \beta \in \mathbb{R}, V \in \mathbb{R}^{+}: \\
\mho_{\beta, V}=\operatorname{span}_{\mathbb{R}}\left\{\omega_{F S}-4 \beta v, v^{0}+\beta \omega_{F S}+\left(V-2 \beta^{2}\right) v\right\} .
\end{gathered}
$$


The parameters $(\beta, V) \in \mathbb{R} \times \mathbb{R}^{+}$of the theories under investigation can be conveniently combined into a parameter $\tau$ on the upper half plane $\mathbb{H}$ :

$$
\tau:=\beta+i \sqrt{\frac{V}{2}} \in \mathbb{H}=\{z \in \mathbb{C} \mid \operatorname{Im}(z)>0\} .
$$

To reproduce Witten's $\mathbb{S}^{2} \simeq \mathbb{C} \cup\{\infty\}$ we now have to divide out all dualities that leave invariant the subvariety of $\widetilde{\mathcal{M}}_{S C F T}^{K 3}$ given by the four-planes $x$ as in (11), and then compactify in a natural way. Recall [AM94; NW01] that all dualities on $\widetilde{\mathcal{M}}_{S C F T}^{K 3}$ are generated by the geometric symmetries, which identify equivalent Einstein metrics, the integral $B$-field shifts $B \mapsto B+\lambda, \lambda \in$ $H^{2}(X, \mathbb{Z})$, and the Nahm-Fourier-Mukai transform $v \leftrightarrow v^{0}$. In our case the geometric symmetries are of no relevance, since we have fixed a specific Einstein metric. The $B$-field shifts are restricted to $B \mapsto B+n \omega_{F S}, n \in \mathbb{Z}$, by our constraint $B \sim \omega_{F S}$. The shift $B \mapsto B+\omega_{F S}$ induces the action

$$
T: \quad \tau \longmapsto \tau+1
$$

on the parameter space $\mathbb{H}$. Finally, to study the Nahm-Fourier-Mukai transform note that with

$$
\beta^{*}:=-\frac{\beta}{V+2 \beta^{2}}, \quad V^{*}:=\frac{V}{\left(V+2 \beta^{2}\right)^{2}},
$$

one has

$$
\mho_{\beta, V}=\operatorname{span}_{\mathbb{R}}\left\{\omega_{F S}-4 \beta^{*} v^{0}, v+\beta^{*} \omega_{F S}+\left(V^{*}-2\left(\beta^{*}\right)^{2}\right) v^{0}\right\} .
$$

So under $v \leftrightarrow v^{0}$ the new parameters are $\left(\beta^{*}, V^{*}\right)$. Hence the Nahm-FourierMukai transform acts on the parameter space $\mathbb{H}$ as

$$
S_{2}: \quad \tau=\beta+i \sqrt{\frac{V}{2}} \longmapsto \frac{-\beta+i \sqrt{V / 2}}{V+2 \beta^{2}}=-\frac{1}{2 \tau} .
$$

Let

$$
\Gamma_{0}(2)_{*}:=\left\langle T, S_{2}\right\rangle,
$$

then Witten's $\mathbb{S}^{2}$ should be realized as a compactification of $\mathbb{H} / \Gamma_{0}(2)_{*}$. It is not hard to show that the fundamental domain of $\Gamma_{0}(2)_{*}$ is

$$
D:=\left\{\left.\tau \in \mathbb{H}|2| \tau\right|^{2} \geq 1,|\operatorname{Re}(\tau)| \leq \frac{1}{2}\right\}
$$

and that $\Gamma_{0}(2)_{*}$ is the normalizer group $\Gamma_{0}(2)_{+}$of $\Gamma_{0}(2)$ in $\mathrm{PSL}_{2}(\mathbb{R}) . T$ identifies the two boundaries of $D$ at $|\operatorname{Re}(\tau)|=\frac{1}{2}$, and $S_{2}$ identifies $\tau$ with $-\bar{\tau}$ on the boundary given by the half circle $2|\tau|^{2}=1$.

\footnotetext{
๙ We cannot resist to use the notation $T$ for the map $\tau \mapsto \tau+1$ and trust that this will cause no confusion with the same notation $T$ for the diffeomorphism type of a real four-torus in the rest of this note.
} 
Our observation that $\Gamma_{0}(2)_{+}$gives the automorphism group of the lattice generated by two null vectors $v^{0}, v$ with $\left\langle v^{0}, v\right\rangle=1$ and $\omega$ with $\langle v, \omega\rangle=$ $\left\langle v^{0}, \omega\right\rangle=0,\langle\omega, \omega\rangle=4$ agrees with [Dol96, Th.7.1]: For $N \in \mathbb{N}, \mathbb{H} / \Gamma_{0}(N)_{+}$is nothing but the moduli space of $M_{N}$ polarized $K 3$-surfaces, i.e. $K 3$-surfaces $X$ which possess $\omega \in \operatorname{Pic}(X)$ with $\langle\omega, \omega\rangle=2 N$.

From (12) it follows that $\mathbb{H} / \Gamma_{0}(2)_{+}$can be naturally compactified by adding $\tau=i \infty$, such that

$$
\mathbb{S}^{2} \simeq \overline{\mathbb{H} / \Gamma_{0}(2)_{+}} .
$$

Thus there are three special points in our $\mathbb{S}^{2}$, given by $\tau=i \infty, \tau=\frac{i}{\sqrt{2}}$, and $\tau=-\frac{1}{2}+\frac{i}{2}$. The monodromies are generated by $T, S_{2}$, and $T S_{2}$, respectively. Hence $\tau=i \infty$ is the point of maximal unipotent monodromy and should give the point $w=\infty$ in $\mathcal{F}_{X}$. Indeed, this is the point we have added to $\mathbb{H} / \Gamma_{0}(2)_{+}$ to compactify, and it corresponds to $V \rightarrow \infty$; (11) shows that our four-plane then degenerates to a plane spanned by $\Omega, \omega_{F S}$, and the null vector $v$. In particular, the plane is independent of the value of $\beta$, as expected. At $\tau=\frac{i}{\sqrt{2}}$ the monodromy has order 2, i.e. gives a Weyl reflection on cohomology as expected around the point $w=1$ in $\mathcal{F}_{X}$ : Note the root $e:=v-v^{0}, e \perp x$, which indeed violates (8). Finally, $\tau=-\frac{1}{2}+\frac{i}{2}$ must correspond to the Gepner point $w=0$. Indeed, the values for volume and $B$-field in this point are $V=\frac{1}{2}$ and $B=-\frac{1}{2} \omega_{F S}$, in agreement with our explicit calculation leading to Prop. 4.

We find a 1: 1 map from our moduli space to Witten's $\mathbb{S}^{2}$ which maps the special points correctly by setting

$$
r+i \vartheta:=2 \pi(\ln (2 V-1)+i \beta), \quad w:=e^{r+i \vartheta} .
$$

The volume enters logarithmically, as expected from the description of the moduli space $\mathcal{M}_{S C F T}^{K 3}$ [AM94; Dij99].

The above analysis gives an independent method to predict the full geometric interpretation of the Gepner model $(2)^{4}$ on the Fermat quartic. It does not use mirror symmetry. However, let us now discuss

\section{Mirror Moonshine on $K 3$}

There is an interesting connection to arithmetic number theory which relates the above analysis to the results of [NS95; LY96], see also [VY00]. Our calculation is independent of the specific complex structure $\Omega_{X}$ as long as $X \subset \mathbb{C P}^{3}$. Hence our family $\mathcal{F}_{X} \simeq \mathbb{S}^{2}$ is the complexified Kähler moduli space of the family of all quartic hypersurfaces in $\mathbb{C P}^{3}$. By mirror symmetry it should agree with the complex structure moduli space of the mirror family, i.e. the family $\left(\mathcal{G}_{z}, z=(4 \psi)^{-4} \in \mathbb{C} \cup\{\infty\}\right)$ of $\mathbb{Z}_{4}^{2}$ orbifolds of the quartic hypersurfaces

$$
z_{0}^{4}+z_{1}^{4}+z_{2}^{4}+z_{3}^{4}-4 \psi z_{0} z_{1} z_{2} z_{3}=0 \quad \text { in } \quad \mathbb{C P}^{3}, \quad \psi \in \mathbb{C} \cup\{\infty\},
$$

where $\mathbb{Z}_{4}^{2}$ is generated by 


$$
\left(z_{0}, z_{1}, z_{2}, z_{3}\right) \longmapsto\left(i z_{0},-i z_{1}, z_{2}, z_{3}\right) ; \quad\left(z_{0}, z_{1}, z_{2}, z_{3}\right) \longmapsto\left(i z_{0}, z_{1},-i z_{2}, z_{3}\right)
$$

and in $\mathcal{G}_{z}$, all quotient singularities coming from fixed points of the $\mathbb{Z}_{4}^{2}$ action are minimally resolved. Here, $z=(4 \psi)^{-4}$ is a true parameter of $\mathcal{G}_{z}$ since $z_{0} \mapsto-i z_{0}$ identifies the surfaces at $\psi$ and $i \psi$.

The quartic (13) with $\psi=1$ has 16 nodes, i.e. is a Kummer surface whose singularities have not been blown up. Hence $\mathcal{G}_{z}$ has monodromy of order 2 around $z=1 / 256$, which therefore corresponds to the point $\tau=\frac{i}{\sqrt{2}}$ in $\mathcal{F}_{X}$. By (11) this means that the $\mathbb{Z}_{4}^{2}$ orbifold of this Kummer quartic has transcendental lattice $\operatorname{diag}(4,2)$, which uniquely determines it by Thm. 1 . The 16 nodes form a single orbit under the $\mathbb{Z}_{4}^{2}$-action, and hence in the $\mathbb{Z}_{4}^{2}$ orbifold give the unique root $e=v-v^{0}$ with $e \perp x$ at $\tau=\frac{i}{\sqrt{2}}(w=1)$ mentioned above. Similarly, around $z=\infty$, the family $\mathcal{G}_{z}$ has monodromy of order 4 . Hence $z=\infty$ corresponds to the Gepner point $\tau=-\frac{1}{2}+\frac{i}{2}$ in $\mathcal{F}_{X}$, and we find that the mirror of $\left(\Sigma_{X_{0}}, V=\frac{1}{2}, B=-\frac{1}{2} \omega_{F S}\right)$ has transcendental lattice $\operatorname{diag}(2,2)$, see $(15)$.

The explicit mirror map for the family of quartics in $\mathbb{C P}^{3}$ has been calculated in [NS95]. There, the group $\Gamma_{0}(2)_{+}$makes its appearance already, and the discussion of its fixed points on $\mathbb{H}$ precisely matches our observations above. In [LY96] these results are rediscovered and extended, and it is pointed out that with $q(\tau):=e^{2 \pi i \tau}, z=z(q)$, the function $\tau \mapsto 1 / z-96$ is the Hauptmodul of $\Gamma_{0}(2)_{+}$. Given the rôle that $\Gamma_{0}(2)_{+}$played in the above analysis, this of course is hardly a surprise.

The occurrence of Hauptmoduln in the mirror map has been entirely demystified by C. Doran [Dor00b; Dor00a]: By the Torelli theorem, the period domain of a one-parameter family of rank 19 lattice polarized $K 3$-surfaces $p: \mathcal{X} \longrightarrow S$ lies on a non-degenerate quadric in $\mathbb{C P}^{2}$. Therefore, generalizing the $j$-function, one can define a functional invariant $\mathcal{H}_{M}: S \longrightarrow \mathbb{K}_{M}$, where $\mathbb{K}_{M}=\Gamma_{M} \backslash D_{M}$ denotes the coarse moduli space of $M$-polarized $K 3$-surfaces, $D_{M}$ its smooth universal covering space, and $\Gamma_{M}$ an arithmetic group. $\mathcal{H}_{M}$ is the composition of the period morphism $S \longrightarrow D_{M}$ and the arithmetic quotient $D_{M} \longrightarrow \mathbb{K}_{M}$. Doran shows that the Picard-Fuchs equation for the family $\mathcal{X}$ is the symmetric square of a second order homogeneous linear Fuchsian ordinary differential equation. This perhaps is related to the existence of SI-structures as in [Pet86], see [VY00, 5.1]. The truncated projective period map gives the projective period ratio $z(q)$ of that square root equation. The latter is the uniformizing differential equation for $S$ iff $\mathcal{H}_{M}$ is branched at most over the orbifold divisor in $\operatorname{Pic}\left(\mathbb{K}_{M}\right)$. Now on the one hand, $z(q)$ gives the mirror map for $\mathcal{X}$, and on the other hand, by uniformization of orbifold Riemann surfaces, $z(q)$ will be an automorphic function for a finite index subgroup of a Fuchsian group of the first kind iff the base curve $S$ is so uniformized. This result even generalizes directly to $n$-parameter families of rank $20-n$ lattice polarized $K 3$-surfaces [Dor00a]. 
Summarizing, the beautiful arithmetic properties of the mirror maps of families of $K 3$-surfaces [LY96] are neither restricted to the genus zero case, nor to the one-parameter case, and they seem to be independent of moonshine.

\section{An application of Shioda-Inose structures?}

Recall the idea underlying our proof of Prop. 3: Since there is an SI-structure $X=\widetilde{T_{0} / \mathbb{Z}_{4}} \stackrel{2: 1}{\longrightarrow} \widetilde{T_{0} / \mathbb{Z}_{2}} \stackrel{2: 1}{\longleftarrow} T_{0}$, we predicted the existence of a $\mathbb{Z}_{2}$-type orbifold $(\widehat{2})^{4}=(2)^{4} / \sigma$ which could be identified with $\mathcal{T}_{0} / \mathbb{Z}_{2}$. However, having found $\sigma=[2,2,0,0], \eta$ with $(\widehat{2})^{4}=(2)^{4} / \sigma$ and $(\widehat{2}) / \eta=(2)^{4}$ does not mean that $\sigma$ is induced by the symplectic orbifold $X=\widetilde{T_{0} / \mathbb{Z}_{4}} \stackrel{2: 1}{\longrightarrow} \widetilde{T_{0} / \mathbb{Z}_{2}}$ from the SIstructure. In fact, in this section we will argue the contrary.

Assume that an SI-structure $X \stackrel{\bmod \iota}{\longrightarrow} Y \stackrel{\bmod }{\longleftarrow} T$ as in Thm. 3 can be lifted to the level of SCFTs. This implies that $X$ and $T$ admit $\iota$ and $\pi$ invariant Einstein metrics specified by $\Sigma_{X} \subset H^{2}(X, \mathbb{R})^{\iota}$ and $\Sigma_{T} \subset H^{2}(T, \mathbb{R})^{\pi}$, respectively. Moreover, $\iota_{*} \Sigma_{X}=\pi_{*} \Sigma_{T} \subset H^{2}(Y, \mathbb{R})$. However, by the analysis of the NikULin InVOLUTiOn $\iota$ performed in [Mor93], $\left(\iota_{*} \Sigma_{X}\right)^{\perp} \cap H^{2}(Y, \mathbb{Z})=$ $N \oplus E_{8}(-1)$, where the lattice $E_{8}(-1)$ has intersection form the negative of the Cartan matrix of $E_{8}$, and $N$ is generated by eight pairwise orthogonal roots $e_{1}, \ldots, e_{8}$ and $\frac{1}{2} \sum_{i=1}^{8} e_{i}$. On the other hand, by classical results on Kummer surfaces, $\left(\pi_{*} \Sigma_{T}\right)^{\perp} \cap H^{2}(Y, \mathbb{Z})=\Pi$ is the KUMMER LATTICE which is not isomorphic to $N \oplus E_{8}(-1)$. Hence $\iota_{*} \Sigma_{X} \neq \pi_{*} \Sigma_{T}$.

This observation can be confirmed by a direct calculation: In [SI77], the branch locus of $X \stackrel{\bmod \iota}{\longrightarrow} Y$ is described explicitly in terms of the lattice $\pi_{*} H^{2}(T, \mathbb{Z}) \oplus \Pi \subset H^{2}(Y, \mathbb{Z})$. In particular, the roots $e_{1}, \ldots, e_{8} \in N$ are determined. They are the irreducible components in the exceptional divisor for the minimal resolution of all singularities in $X / \iota$. Then the map $\widetilde{\eta}$ reversing the orbifold by $\iota$ should act as $\widetilde{\eta}_{\mid N}=-$ id, $\widetilde{\eta}_{\mid N^{\perp}}=$ id. Hence with the results of [SI77], $\widetilde{\eta}$ can be calculated explicitly. One checks that this map only leaves a subspace of signature $(2,3)$ of $\pi_{*} H^{2}(T, \mathbb{R})$ invariant, such that no Einstein metric $\Sigma_{T} \subset H^{2}(T, \mathbb{R})^{\pi}$ can be found with $\pi_{*} \Sigma_{T} \subset H^{2}(Y, \mathbb{R})^{\tilde{\eta}}$. In other words, $\widetilde{\eta}$ does not extend to a symmetry of the $\mathbb{Z}_{2}$ orbifold $\mathcal{T} / \mathbb{Z}_{2}$ of any toroidal theory $\mathcal{T}$.

Summarizing, the symmetry $\sigma=[2,2,0,0]$ of $(2)^{4}$ which was used in Sect. 2 is not induced by the Nikulin involution $\iota$ of the SI-structure $\widetilde{T_{0} / \mathbb{Z}_{4}} \stackrel{\bmod }{\longrightarrow}$ $\widetilde{T_{0} / \mathbb{Z}_{2}} \stackrel{\bmod \pi}{\longleftarrow} T_{0}$. But recall that in Prop. 4 we proved that $(2)^{4}$ also has a geometric interpretation on the Fermat quartic $X_{0}$. Gepner had conjectured this [Gep87; Gep88] on the basis of a comparison between symmetries of $(2)^{4}$ and symplectic automorphisms of the quartic $X_{0}$. Witten's analysis [Wit93] extends these ideas and provides us with a cross-check for our results in Sect. 3. Under these identifications, $\sigma=[2,2,0,0]$ corresponds to the symplectic automorphism 


$$
\sigma: \quad\left(z_{0}, z_{1}, z_{2}, z_{3}\right) \longmapsto\left(-z_{0},-z_{1}, z_{2}, z_{3}\right) \text { in } \mathbb{C P}^{3} .
$$

Hence $(\widehat{2})^{4}=(2)^{4} / \sigma$ can be expected to admit a geometric interpretation on $\widetilde{X_{0} / \sigma}$. The following theorem implies that we have already proved this:

Theorem 4. [Ino76, Thms.1,2] An attractive K3-surface $X$ with associated quadratic form $Q_{X}$ is biholomorphic to a quartic surface

$$
X\left(f_{1}, f_{2}\right): \quad f_{1}\left(z_{0}, z_{1}\right)+f_{2}\left(z_{2}, z_{3}\right)=0 \quad \text { in } \quad \mathbb{C P}^{3}
$$

iff $X$ is very attractive, i.e. by Def. 2 iff $Q_{X}=4 Q_{T}$ for some even integral positive definite quadratic form $Q_{T}$. In that case, let $Y=\widetilde{X / \sigma}$. Then $Y$ is an attractive K3-surface with associated quadratic form $Q_{Y}$ such that $Q_{X}=$ $2 Q_{Y}$. Moreover, $Y$ is canonically biholomorphic to the Kummer surface $\widetilde{T / \mathbb{Z}_{2}}$ where $T=E_{1} \times E_{2}$ with $E_{k}: z_{2}^{2}=f_{k}\left(z_{0}, z_{1}\right)$ in $\mathbb{C P}_{(1,1,2)}^{2}$ is an attractive torus with associated quadratic form $Q_{T}$.

Indeed, since $Q_{X_{0}}=4 Q_{T_{0}}$, Thm. 4 implies that $\widetilde{X_{0} / \sigma} \simeq \widetilde{T_{0} / \mathbb{Z}_{2}}$, which by Prop. 2 extends to $(\widehat{2})^{4}=(2)^{4} / \sigma=\mathcal{T}_{0} / \mathbb{Z}_{2}$ on the level of SCFTs. In other words, our proof that $(2)^{4}=\mathcal{T}_{0} / \mathbb{Z}_{4}$, which relied on the chain of orbifolds $(2)^{4} \stackrel{\bmod \sigma}{\longrightarrow}(\widehat{2})^{4} \stackrel{\bmod \eta}{\longrightarrow}(2)^{4}$, geometrically translates into $Y=\widetilde{X_{0} / \sigma} \simeq \widetilde{T_{0} / \mathbb{Z}_{2}}$, $\widehat{Y / \eta} \simeq \widetilde{T_{0} / \mathbb{Z}_{4}}$. On the level of attractive complex structures we observe that the associated quadratic forms transform as

$$
Q_{X_{0}}=\operatorname{diag}(8,8) \stackrel{\bmod }{\longrightarrow} \sigma Q_{\widetilde{T_{0} / \mathbb{Z}_{2}}}=\operatorname{diag}(4,4) \stackrel{\bmod \eta}{\longrightarrow} Q_{\widetilde{T_{0} / \mathbb{Z}_{4}}}=\operatorname{diag}(2,2) .
$$

Now recall from our discussion in Sect. 3 that $(2)^{4}$ should be given by a fourplane $x_{(2)^{4}} \in \mathcal{M}_{S C F T}^{K 3}$ with $x_{(2)^{4}}=\Omega_{X_{0}} \oplus \mho_{-\frac{1}{2}, \frac{1}{2}}$, where $\Omega_{X_{0}}, \mho^{*}:=\mho_{-\frac{1}{2}, \frac{1}{2}}$ are generated by lattice vectors in $H^{\text {even }}(X, \mathbb{Z})$ such that by $(11)$

$$
\mho^{*}=\operatorname{span}_{\mathbb{R}}\left\{\omega_{F S}+2 v, v^{0}-\frac{1}{2} \omega_{F S}\right\}=\operatorname{span}_{\mathbb{R}}\left\{\omega_{F S}+v-v^{0}, v+v^{0}\right\} .
$$

Hence the associated quadratic forms are $Q_{X_{0}}=\operatorname{diag}(8,8)$ and $Q_{\mho^{*}}=$ $\operatorname{diag}(2,2)$, which are exchanged in our chain (14). Finally, observe that the four-plane $x_{(\widehat{2})^{4}} \in \mathcal{M}_{S C F T}^{K 3}$ corresponding to $(\widehat{2})^{4}=(2)^{4} / \sigma$ can be split into $x_{(\widehat{2})^{4}}=\widehat{\Omega} \oplus \widehat{\mho}$, where $\widehat{\Omega}, \widehat{\mho}$ are generated by lattice vectors in $H^{\text {even }}(X, \mathbb{Z})$ such that the associated quadratic forms are $Q_{\widehat{\Omega}}=\operatorname{diag}(4,4)$ and $Q_{\widehat{\mho}}=\operatorname{diag}(4,4)$. Our identification $(\widehat{2})^{4}=(2)^{4} / \sigma=\mathcal{T}_{0} / \mathbb{Z}_{2}$ is blind towards an exchange $\widehat{\Omega} \leftrightarrow \widehat{\mho}$, so our chain (14) schematically corresponds to $\Omega_{X_{0}} \oplus \mho^{*} \rightarrow \widehat{\Omega} \oplus \widehat{\mho} \sim \widehat{\mho} \oplus \widehat{\Omega} \rightarrow \mho^{*} \oplus \Omega_{X_{0}}$.

The above implies a possible generalization of our construction: By Thms. 4, for $a, b, c \in \mathbb{Z}$ with $a>0$ and $4 a c-b^{2}>0$, there is a very attractive quartic $X_{a, b, c} \subset \mathbb{C P}^{3}$ with quadratic form on the transcendental lattice given by

$$
Q_{a, b, c}=\left(\begin{array}{ll}
8 a & 4 b \\
4 b & 8 c
\end{array}\right) .
$$


On the other hand, our discussion in Sect. 3 was independent of the specific complex structure $\Omega_{a, b, c}=\Omega_{1,0,1}$ on the quartic hypersurface in $\mathbb{C P}^{3}$ which we chose in order to investigate $(2)^{4}$. Hence for the family $\mathcal{F}_{X_{a, b, c}} \simeq \overline{\mathbb{H} / \Gamma_{0}(2)_{+}}$of $N=(4,4)$ SCFTs on $X_{a, b, c}$, equipped with the normalized Kähler class $\omega_{F S}$ of the Fubini-Study metric and with $B$-field $B=\beta \omega_{F S}$, the discussion in Sect. 3 shows that there is a point with monodromy of order 4 at $\beta=-\frac{1}{2}, V=\frac{1}{2}$. This point corresponds to a SCFT with $x_{a, b, c}=\Omega_{a, b, c} \oplus \mho^{*} \in \mathcal{M}_{S C F T}^{K 3}$ where $\Omega_{a, b, c}, \mho^{*}$ are generated by lattice vectors such that the associated quadratic forms are $Q_{a, b, c}$ and $Q_{\mho^{*}}=\operatorname{diag}(2,2)$. Now recall from Cor. 1 that up to $\Omega_{a, b, c} \leftrightarrow \mho^{*}$ these are the data that can be obtained from a $\mathbb{Z}_{4}$ orbifold construction. If $x_{a, b, c}$ gives the location of $\mathcal{T}_{a, b, c} / \mathbb{Z}_{4}$ in $\mathcal{M}_{S C F T}^{K 3}$, a chain similar to (14) should exist. Indeed,

Proposition 5. Let $a, b, c \in \mathbb{Z}$ such that $\mathcal{T}_{a, b, c}$ denotes the toroidal SCFT specified in Cor. 1. Namely, $\mathcal{C}_{a, b, c}=\mathcal{T}_{a, b, c} / \mathbb{Z}_{4}$ is given by $x_{a, b, c}=\Omega^{*} \oplus$ $\mho_{a, b, c} \in \mathcal{M}_{S C F T}^{K 3}$, where $\Omega^{*} \cap H^{\text {even }}(X, \mathbb{Z})$ and $\mho_{a, b, c} \cap H^{\text {even }}(X, \mathbb{Z})$ have rank 2 and associated quadratic forms $Q_{\Omega^{*}}=\operatorname{diag}(2,2)$ and $Q_{\mho_{a, b, c}}=Q_{a, b, c}$ as in (16), respectively. Then $\mathcal{C}_{a, b, c}$ admits a geometric interpretation on the very attractive K3-surface $X_{a, b, c}$ with associated quadratic form $Q_{a, b, c}$, normalized Kähler class $\omega_{Q} \in H^{2}\left(X_{a, b, c}, \mathbb{Z}\right)$ with $\left\langle\omega_{Q}, \omega_{Q}\right\rangle=4$, volume $V=\frac{1}{2}$, and $B$-field $B=-\frac{1}{2} \omega_{Q}$.

Proof. The proof is entirely analogous to the proof of Prop. 4. We use the notations and results of the proof of Cor. 1 . Hence $x_{a, b, c}=\Omega^{*} \oplus \mho_{a, b, c}$ with

$$
\begin{aligned}
\Omega^{*} \cap H^{\text {even }}(X, \mathbb{Z}) & =\operatorname{span}_{\mathbb{Z}}\left\{\widetilde{\Omega}_{1}, \widetilde{\Omega}_{2}\right\}, \\
\mho_{a, b, c} \cap H^{\text {even }}(X, \mathbb{Z}) & =\operatorname{span}_{\mathbb{Z}}\left\{\xi_{1}=\widetilde{\omega}_{a}+b \widehat{v}, \xi_{2}=4 \widehat{v}^{0}+\check{B}_{4}+(c+4) \widehat{v}\right\} .
\end{aligned}
$$

If $\left(\lambda_{1}, \ldots, \lambda_{4}\right)$ denotes a $\mathbb{Z}$-basis of $\Lambda$ for $T=\mathbb{R}^{4} / \Lambda$, which is dual to the basis $\left(\mu_{1}, \ldots, \mu_{4}\right)$ of $\Lambda^{*}$ used in the proof of Cor. 1 , then the $\mathbb{Z}_{4}$-action $\zeta_{4}$ on $T$ has fixed points at each $\sum_{k} \frac{i_{k}}{2} \lambda_{k}$ with $i_{k} \in\{0,1\}$. By the results of [NW01], there are $e_{\left(i_{1}, i_{2}, i_{3}, i_{4}\right)} \in H^{2}(X, \mathbb{Z}) \cap\left(\pi_{*} H^{2}(X, \mathbb{Z})^{\mathbb{Z}_{4}}\right)^{\perp}$ with $\left\langle e_{\left(i_{1}, i_{2}, i_{3}, i_{4}\right)}, e_{\left(i_{1}, i_{2}, i_{3}, i_{4}\right)}\right\rangle=$ -2 and $\left\langle e_{\left(i_{1}, i_{2}, i_{3}, i_{4}\right)}, \check{B}_{4}\right\rangle=2$ (Poincaré dual to classes in the exceptional divisor of the blow up of $\left.\sum_{k} \frac{i_{k}}{2} \lambda_{k}\right)$ such that

$$
\begin{aligned}
& v_{Q}^{0}:=\frac{1}{2}\left(\widetilde{\Omega}_{1}+\widetilde{\Omega}_{2}\right)+\frac{1}{2}\left(e_{(0,1,0,1)}-e_{(0,1,1,0)}\right), \\
& v_{Q}:=\frac{1}{2}\left(\widetilde{\Omega}_{1}-\widetilde{\Omega}_{2}\right)-\frac{1}{2}\left(e_{(0,1,0,1)}-e_{(0,1,1,0)}\right)
\end{aligned}
$$

are lattice vectors. One checks that $\left(v_{Q}^{0}, v_{Q}\right)$ obey $(6)$; in fact, $\left(v_{Q}^{0}, v_{Q}\right)$ agree with the vectors used in the proof of Prop. 4, see [NW01, (2.18)]. To determine the decomposition (10), we observe that $\xi\left(\Sigma_{Q}\right)=v_{Q}^{\perp} \cap x_{a, b, c}$ is generated by $\xi\left(\omega_{Q}\right):=\widetilde{\Omega}_{1}+\widetilde{\Omega}_{2}, \xi_{1}, \xi_{2}$. Moreover, $\xi_{k} \perp v_{Q}^{0}$ for $k \in\{1,2\}$, and these vectors generate a two-plane $\Omega_{Q} \subset \Sigma_{Q}$ such that $\Omega_{Q} \cap H^{\text {even }}(X, \mathbb{Z})$ has quadratic 
form $Q_{a, b, c}$. By Thm. 4 this means that we obtain a geometric interpretation of $\mathcal{C}_{a, b, c}=\mathcal{T}_{a, b, c} / \mathbb{Z}_{4}$ on $X_{a, b, c}$. Finally,

$$
\omega_{Q}=\xi\left(\omega_{Q}\right)-\left\langle\xi\left(\omega_{Q}\right), v_{Q}^{0}\right\rangle v_{Q}=2 \widetilde{\Omega}_{2}+e_{(0,1,0,1)}-e_{(0,1,1,0)} \in H^{\text {even }}(X, \mathbb{Z})
$$

obeys $\left\langle\omega_{Q}, \omega_{Q}\right\rangle=4$, and $\xi\left(\Sigma_{Q}\right)^{\perp} \cap x_{a, b, c}$ is generated by $\xi_{4}=\frac{1}{2}\left(\widetilde{\Omega}_{1}-\widetilde{\Omega}_{2}\right)=$ $v_{Q}^{0}-\frac{1}{2} \omega_{Q}$, as claimed.

By results of [SI77] we know that each quartic is biholomorphic to a Kummer surface, if it is very attractive. Since all orbifolds of toroidal SCFTs can be constructed explicitly, this means that for every quartic $X \subset \mathbb{C P}^{3}$ we can find a $\mathbb{Z}_{2}$ orbifold CFT $\mathcal{T}_{X}^{\prime} / \mathbb{Z}_{2}$ of a toroidal SCFT which admits a geometric interpretation on $X$, if $X$ is very attractive. Prop. 5 states that we then can also find a $\mathbb{Z}_{4}$ orbifold CFT $\mathcal{T}_{X} / \mathbb{Z}_{4}$ which has geometric interpretation with the same complex structure, but in general will be different from $\mathcal{T}_{X}^{\prime} / \mathbb{Z}_{2}$ : The normalized Kähler classes, volumes, and $B$-fields will disagree. Note, e.g., that $(2)^{4}$ does not agree with any $\mathbb{Z}_{2}$ orbifold of a toroidal SCFT [NW01, Sect.2.4]. The arguments used in Sect. 3 as well as the result of Prop. 5 indicate that, as opposed to the $\mathbb{Z}_{2}$ orbifold model $\mathcal{T}_{X}^{\prime} / \mathbb{Z}_{2}$, the $\mathbb{Z}_{4}$ orbifold $\mathcal{T}_{X} / \mathbb{Z}_{4}$ may give a model on the very attractive quartic $X$ with the natural hyperkähler structure:

Conjecture 1. The two-plane $\Omega^{*}$ in Prop. 5 agrees with (15), i.e. we can find a geometric interpretation of $\mathcal{C}_{a, b, c}$ on $X_{a, b, c}$ with normalized Kähler class the class $\omega_{F S}$ of the Fubini-Study metric in $\mathbb{C P}^{3}$. In other words, we can construct an $N=(4,4)$ SCFT with $c=6$ on every very attractive quartic, equipped with its natural hyperkähler structure.

To sustain Conj. 1 recall that the analysis of Sect. 3 predicts the model at $w=0$ in $\mathcal{F}_{X_{a, b, c}}$ to have parameters $V=\frac{1}{2}, B=-\frac{1}{2} \omega_{F S}$. The monodromy around $w=0$ has order 4 , which is consistent with the model having a $\mathbb{Z}_{4}$ orbifold interpretation $\mathcal{T}_{a, b, c} / \mathbb{Z}_{4}$ as in Prop. 5. In fact, assume that $(2)^{4}$ has a geometric interpretation $\left(\Sigma_{X_{0}}, V=\frac{1}{2}, B=-\frac{1}{2} \omega_{F S}\right)$ as implied by [Wit93]. The Fermat quartic $X_{0} \subset \mathbb{C P}^{3}$ possesses a group $G \cong\left(\mathbb{Z}_{4}^{3} \rtimes \mathcal{S}_{4}\right) / \mathbb{Z}_{4}$ of symplectic automorphisms with Mukai number $\mu(G)=5$ (see [Muk88; Asp95]), which also leaves $\omega_{F S}$ invariant. This means that $\operatorname{dim}\left(H^{2}\left(X_{0}, \mathbb{R}\right)^{G}\right)=3$ and hence $\Sigma_{X_{0}}=H^{2}\left(X_{0}, \mathbb{R}\right)^{G}$, as argued in [Asp95]. On the other hand, Thms. 1, 2 and Prop. 5 imply $x_{(2)^{4}}=\Omega_{X_{0}} \oplus \mho$ with $\Omega_{X_{0}}=\mho_{1,0,1}$, hence $\mho=\Omega^{*}$. Although this does not mean that the geometric interpretation of $(2)^{4}$ in Props. 4,5 agrees with the "true quartic" one, i.e. that $v^{0}=v_{Q}^{0}, v=v_{Q}$ with $v^{0}, v$ as in (15), our assumptions ensure that a "true quartic" interpretation by $v^{0}, v$ exists. The analysis of [Wit93] also implies an identification of some of the deformations of the SCFT $(2)^{4}$ with polynomial deformations of the Fermat quartic $X_{0}$ as in [Gep87]. Namely, identifying $\left(1_{ \pm 10}^{ \pm 10}\right)$ in the $(j+1)^{\text {st }}$ component of $(2)^{4}$ with $\alpha z_{j}, \alpha \in \mathbb{C}$,

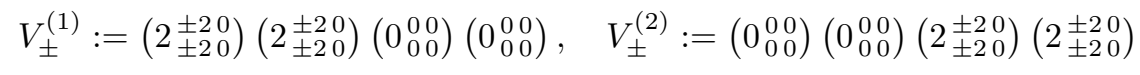


correspond to deformations of $X_{0}$ as in (2) by $\alpha_{1} z_{0}^{2} z_{1}^{2}, \alpha_{2} z_{2}^{2} z_{3}^{2}$ with $\alpha_{k} \in \mathbb{C}$.

The $V_{ \pm}^{(k)}$ are those $\left(\frac{1}{2}, \frac{1}{2}\right)$-fields which $(2)^{4}$ shares with $(2)^{2} \otimes(2)^{2}$ and one of its subtheories $(2)^{2}$. Since $(2)^{2}$ is the toroidal SCFT on $\mathbb{R}^{2} / \mathbb{Z}^{2}$ with vanishing $B$-field, we have $(2)^{2} \otimes(2)^{2}=\mathcal{T}_{0}$, and Prop. 3 implies $(2)^{4}=$ $(2)^{2} \otimes(2)^{2} / \mathbb{Z}_{4}$. Hence $V_{ \pm}^{(k)}$ give those $\mathbb{Z}_{4}$-invariant deformations in $\mathcal{T}_{0}$ which come from deformations of one of the subtheories $(2)^{2}$, i.e. deformations of the radii $R_{k}$ of $E_{k}, k \in\{1,2\}$, in Cor. 1, and $B$-field deformations in $\Omega_{T_{0}}^{\perp} \cap$ $H^{2}\left(T_{0}, \mathbb{Z}\right)^{\mathbb{Z}_{4}}$. In view of the four-plane $x_{(2)^{4}}$ with notations as in $(17)$, the volume-deformation of $T_{0}$ corresponds to a deformation of $\xi_{2}$ by $\delta V:=\widehat{v}$, and the deformation of $R_{2} / R_{1}$ corresponds to a deformation of $\xi_{1}$ by $\delta \widetilde{\omega}:=\widetilde{\omega}_{2}-\widetilde{\omega}_{1}$. Similarly, all $V_{ \pm}^{(k)}$ give deformations of $\xi_{1}, \xi_{2}$ by $\delta V, \delta \widetilde{\omega}$, respectively.

To show that Conj. 1 holds in general if the above assumptions hold for $(2)^{4}$, first note that in Prop. 5 we use the same $\Omega^{*}$ for all $a, b, c$. We can maintain the "true quartic" geometric interpretation with $v^{0}, v$ as in (15) for all $a, b, c$, if $v^{0}$ and $v$ (with $v^{0}, v \perp \mho_{1,0,1}$ ) are orthogonal to all $\mho_{a, b, c}$, i.e. to $\widehat{v}=\delta V$ and $\delta \widetilde{\omega}$. But the latter follows from the fact that $\delta V$ and $\delta \widetilde{\omega}$ give polynomial deformations of $X_{0}$ as in (2) by complex multiples of $z_{0}^{2} z_{1}^{2}, z_{2}^{2} z_{3}^{2}$, i.e. deformations of the complex structure in the quartic interpretation (15) of $(2)^{4}$. Note that these polynomial deformations are also compatible with the form $X\left(f_{1}, f_{2}\right): f_{1}\left(z_{0}, z_{1}\right)+f_{2}\left(z_{2}, z_{3}\right)=0$ in $\mathbb{C P}^{3}$ of every very attractive quartic as in Thm. 4.

\section{Discussion}

We hope to have convinced the reader that the investigation of SCFTs associated to $K 3$ remains an interesting and challenging enterprize. The emphasis of this work lies on a utilization of results in number theory and geometry, specifically the SHIODA-INOSE-STRUCTURES [SM74; SI77; Ino76; Mor93]. That these structures should be useful in the context of string theory had already been noticed in [Mooa; Moob].

Another deep and interesting connection between number theory and SCFTs on $K 3$ is the so-called MIRROR MOONSHINE PHENOMENON [LY96; VY00; Dor00a; Dor00b]. By a careful application of Witten's analysis of phases in supersymmetric gauge theories [Wit93] to the $K 3$-case we confirm that the FRICKE MODULAR GROUP $\Gamma_{0}(2)+$ makes a natural appearance in the study of the Fermat quartic $X_{0} \subset \mathbb{C P}^{3}$. In particular, this gives a simple independent method to predict $B$-field and volume of the quartic interpretation of the Gepner model $(2)^{4}$. This method is also easily applied to the Gepner model $(4)^{3}$, which should admit a geometric interpretation on the Fermat hypersurface in $\mathbb{C P}_{(1,1,1,3)}^{3}$. It would be interesting to extend these ideas to more-parameter cases. Since Doran's analysis of the mirror moonshine phenomenon allows such an extension, we hope that this will be possible.

Though the idea of inverting Shioda-Inose structures by appropriate orbifold constructions is the guiding principle for our proof [NW01, Thm.2.13] 
that the Gepner model $(2)^{4}$ agrees with a $\mathbb{Z}_{4}$ orbifold, $(2)^{4}=\mathcal{T}_{0} / \mathbb{Z}_{4}$, we have not succeeded to perform such an inversion in general. In fact, we have shown that such an inversion is impossible, as long as one works with a fixed geometric interpretation, that is a fixed choice of grading in $H^{\text {even }}(X, \mathbb{R})$. Since Shioda-Inose structures exist as geometric constructions for arbitrary attractive $K 3$-surfaces, the original idea suggested that a change of geometric interpretation should not be necessary. Whether a combination of orbifold constructions and T-dualities can be found which leads to an inversion of Shioda-Inose structures, thereby giving SCFTs associated to arbitrary attractive $K 3$-surfaces, is left as an open problem for future work.

However, we have succeeded to generalize our method of proof for $(2)^{4}=$ $\mathcal{T}_{0} / \mathbb{Z}_{4}$ in a different direction. Namely, for every very attractive quartic $X$ we find a $\mathbb{Z}_{4}$ orbifold CFT $\mathcal{T}_{X} / \mathbb{Z}_{4}$ which admits a geometric interpretation on $X$. We conjecture that it even allows a geometric interpretation that carries the natural hyperkähler structure which is induced by the Fubini-Study metric on $\mathbb{C P}^{3}$. In fact, we argue that if this conjecture is true for $(2)^{4}$ and the Fermat quartic, where it is generally believed, it should hold for all very attractive quartics. As a next step, our methods should imply that $\mathbb{Z}_{4}$ orbifolds can be used to construct SCFTs with geometric interpretation on non-attractive quartics $X\left(f_{1}, f_{2}\right)$ of the form $f_{1}\left(z_{0}, z_{1}\right)+f_{2}\left(z_{2}, z_{3}\right)=0$ in $\mathbb{C P}^{3}$. The details are left for a future publication.

\section{References}

[AM94] P.S. Aspinwall And D.R. MorRison, String theory on K3 surfaces, in: Mirror symmetry, B. Greene and S.T. Yau, eds., vol. II, 1994, pp. 703-716; hep-th/9404151.

[Asp95] P.S. AsPINWALL, Enhanced gauge symmetries and K3-surfaces, Phys. Lett. B357 (1995), 329-334; hep-th/9507012.

[Asp97] _ K3-surfaces and string duality, in: Fields, strings and duality (Boulder, CO, 1996), World Sci. Publishing, River Edge, NJ, 1997, pp. 421-540; hep-th/9611137.

[BPdV84] W. Barth, C. Peters, and A. Van de Ven, Compact Complex Surfaces, Springer-Verlag, Berlin Heidelberg New York Tokyo, 1984.

[Cec91] S. Cecotti, N=2 Landau-Ginzburg vs. Calabi-Yau $\sigma$-models: Non-perturbative aspects, Int. J. Mod. Phys. A6 (1991), 17491813.

[COGP91] P. Candelas, X.C. De La Ossa, P.S. Green, and L. Parkes, A pair of Calabi-Yau manifolds as an exactly soluble superconformal theory, Nucl. Phys. B359 (1991), 21-74.

[Dij99] R. DiJKGRAAF, Instanton strings and hyperkaehler geometry, Nucl. Phys. B543 (1999), 545-571; hep-th/9810210. 
[Dol96] I.V. Dolgachev, Mirror symmetry for lattice polarized K3 surfaces. Algebraic geometry, 4, J. Math. Sci. 81 (1996), 2599-2630; alg- geom/9502005.

[Dor00a] C. DoRAn, Picard-Fuchs uniformization and modularity of the mirror map, Commun. Math. Phys. 212 (2000), no. 3, 625-647.

[Dor00b] —, Picard-Fuchs uniformization: modularity of the mirror map and mirror-moonshine, in: The arithmetic and geometry of algebraic cycles (Banff, AB, 1998), vol. 24 of CRM Proc. Lecture Notes, Amer. Math. Soc., Providence, RI, 2000, pp. 257-281; math.ag/9812162.

[EOTY89] T. Eguchi, H. Ooguri, A. Taormina, And S.-K. Yang, Superconformal algebras and string compactification on manifolds with SU(n) holonomy, Nucl. Phys. B315 (1989), 193-221.

[Gep87] D. GePner, Exactly solvable string compactifications on manifolds of $S U(N)$ holonomy, Phys. Lett. 199B (1987), 380-388.

[Gep88] - Space-time supersymmetry in compactified string theory and superconformal models, Nucl. Phys. B296 (1988), 757-778.

[GP90] B.R. Greene And M.R. Plesser, Duality in Calabi-Yau moduli space, Nucl. Phys. B338 (1990), 15-37.

[Ino76] H. Inose, On certain Kummer surfaces which can be realized as non-singular quartic surfaces in $\mathbb{P}^{3}$, J. Fac. Sci. Univ. Tokyo Sec. IA 23 (1976), 545-560.

[LVW89] W. Lerche, C. VAFA, AND N.P. WARner, Chiral rings in $N=2$ superconformal theories, Nucl. Phys. B324 (1989), 427-474.

[LY96] B.H. Lian AND S.-T. YAU, Arithmetic properties of mirror map and quantum coupling, Commun. Math. Phys. 176 (1996), 163192; hep- th/9411234.

[Mooa] G.W. Moore, Arithmetic and attractors; hep-th/9807087.

[Moob] —, Attractors and arithmetic; hep-th/9807056.

[Mor93] D.R. MORRISON, Mirror symmetry and rational curves on quintic threefolds: a guide for mathematicians, J. Amer. Math. Soc. 6 (1993), no. 1, 223-247; alg-geom/9202004.

[Muk88] S. MuKaI, Finite groups of automorphisms of K3 surfaces and the Mathieu group, Invent. Math. 94 (1988), 183-221.

[Nar86] K.S. NARAIN, New heterotic string theories in uncompactified dimensions < 10, Phys. Lett. 169B (1986), 41-46.

[NS95] M. Nagura And K. SugiYama, Mirror symmetry of K3 and torus, Int. J. Mod. Phys. A10 (1995), 233-252; hep-th/9312159.

[NW01] W. NAhm AND K. Wendland, A hiker's guide to K3 - Aspects of $N=(4,4)$ superconformal field theory with central charge $c=6$, Commun. Math. Phys. 216 (2001), 85-138; hep-th/9912067.

[Pet86] C. Peters, Monodromy and Picard-Fuchs equations for families of K3-surfaces and elliptic curves, Ann. Sci. École Norm. Sup. 19 (1986), no. 4, 583-607. 
[Sei88] N. SEIBERG, Observations on the moduli space of superconformal field theories, Nucl. Phys. B303 (1988), 286-304.

[SI77] T. Shioda And H. InOse, On singular K3 surfaces, in: Complex Analysis and Algebraic Geometry, W.L. Bailey and T. Shioda, eds., Cambridge Univ. Press, 1977, pp. 119-136.

[SM74] T.J. Shioda And N. MitAni, Singular abelian surfaces and binary quadratic forms, in: Classification of Algebraic Varieties and Compact Complex Manifolds, A. Dold and B. Eckmann, eds., Lecture Notes in Math. 412, 1974, pp. 259-287.

[VY00] H. VERRILl AND N. Yui, Thompson series, and the mirror maps of pencils of K3 surfaces, in: The arithmetic and geometry of algebraic cycles (Banff, AB, 1998), Amer. Math. Soc., Providence, RI, 2000, pp. 399-432.

[Wen01] K. WENDLAND, Consistency of orbifold conformal field theories on K3, Adv. Theor. Math. Phys. 5 (2001), no. 3, 429-456; hep-th/0010281.

[Wen02] —, Orbifold constructions of K3: A link between conformal field theory and geometry, in: Orbifolds in Mathematics and Physics, AMS series Contemporary Mathematics, Providence R.I., 2002, pp. 333-358; hep-th/0112006.

[Wit93] E. Witten, Phases of $N=2$ theories in two dimensions, Nucl. Phys. B403 (1993), 159-222; hep-th/9301042.

[Wit95] — String theory dynamics in various dimensions, Nucl. Phys. B443 (1995), 85-126; hep-th/9503124. 